\title{
OP-Organisation und OP-Koordination
}

\author{
Theo Smits, Guido Schüpfer, Reto Babst
}

\section{Zusammenfassung}

Der kostenintensive Operationssaalbetrieb muss im Rahmen der gesamten Wertschöpfungskette von chirurgischen Behandlungen optimiert werden, ohne dass Qualitätsstandards leiden. Durch das Zusammentreffen verschiedenster Berufsgruppen mit unterschiedlichsten Professionskulturen sind Interessen und Prioritäten oft unterschiedlich gelagert. Basierend auf einer Ist-Analyse des Operationsprozesses dient ein Operationssaalstatut der Regelung des ganzen Prozesses. Für das Controlling müssen die entsprechenden Daten erhoben und bewertet werden. Notwendig ist daher der Einsatz geeigneter Softwarewerk- zeuge. Schnittstellenprobleme können so erkannt und Lösungen datenbasiert und schrittweise (Change-Management) angegangen werden. Faktoren wie Patientensicherheit, Patientenund Mitarbeiterzufriedenheit müssen parallel dazu erfasst werden, um nicht nur einen effizienten, sondern auch qualitativ hochstehenden Operationsbetrieb zu gewährleisten. Voraussetzung für eine erfolgreiche Änderung einer bestehenden OP-Organisation ist der Wille bzw. die Einsicht aller Beteiligten, dass zielgerichtete Veränderungen zur Effizienzsteigerung führen. Das Primat der Patientensicherheit, der Patienten- und Mitarbeiterzufriedenheit muss jedoch mit Nachhaltigkeit oberstes Gebot bleiben $[3,4,5]$.

\section{Einleitung}

Im Operationssaal arbeiten unterschiedliche Berufsgruppen mit wechselnder Autorität und unterschiedlicher Hierarchie zusammen. DRG basierte Fallabgeltungen erfordern eine Beherrschung der Wertschöpfungskette von Behandlungen auch im Operationssaalbetrieb.

Operationssäle sind oft dezentral gelegen - möglichst nahe im Bereich der Kliniken, welche diese benutzen. Die Operationsprogramme werden vielfach noch dezentral von den Kliniken erstellt. Da keine Absprachen stattfinden, kann ungenutzte Vorhalte- oder Elektivkapazität nicht von anderen genutzt werden. Eine kontinuierliche auf den Patienten bezogene Datenerfassung von der Operationsplanung bis zur Entlassung als Basis für die Vernetzung der verschiedenen Leistungserbringer im Patientenprozess ist meist nicht oder nur inkonstant vorhanden.

OP-JOURNAL 2005; 21: 10-14

(c) Georg Thieme Verlag KG Stuttgart · New York
Planungspannen und Improvisationen führen $\mathrm{zu}$ internen und externen Kommunikationsproblemen mit entsprechendem Konfliktpotenzial.

Mit der zunehmenden Definition von Patientenpfaden haben sich die entscheidenden Schnittstellen zwischen den Sprechstunden, wo Operationsindikationen gestellt werden und den Leistungsstellen, welche die Patientenaufnahme zur Operation koordinieren (Bettendisposition, Bettenstationen, Tageskliniken), zur Anästhesie, zur Radiologie und zu den internistischen Fächern in vielen Kliniken geklärt. Die chirurgische Behandlung eines Patienten kann so funktional in ein Ablaufsystem eingeplant und koordiniert werden $[2,7]$.

\section{Modelle von Operationsorganisationen}

Grundvoraussetzung für den Aufbau einer funktionierenden OP-Organisation ist die Implementierung von erkennbaren und verbindlichen Verantwortungsbereichen, auf denen Entscheidungen gefällt und durchgesetzt werden können.
Dazu gibt es verschiedene Lösungsansätze:

1. Mit herkömmlichen Strukturen, wobei die Operationsabteilung meistens unter der Leitung einer Klinik oder eines Fachbereiches steht und die anderen Kliniken oder Fachbereiche auf die OP-Ressourcen zugreifen können (entspricht einer dezentrale Organisationsform).

2. Interdisziplinäre OP-Lenkungsgruppe (Mischorganisationsform) und OP-Koordination

3. Operationsmanager (entspricht eine zentrale Organisationsform)

Dezentrale Organisationsformen sind häufig das Resultat einer historisch gewachsenen Organisation. Sowohl der Arztdienst der Chirurgie und der Anästhesie, wie auch der OP- und Anästhesiepflegedienst sind oft unterschiedlich organisatorisch strukturiert und involviert.

- Die Übertragung der Verantwortung einer Lenkungsgruppe bestehend z.B. aus einem Chirurgen, einem Anästhesisten, einem Vertreter aus der Operations- und Anästhesiepflege hat den Vorteil, dass alle entscheidenden Berufsgruppen involviert werden. Entsprechend können die Entscheidungswege manchmal verzögert sein. Die Vorgabe einer Geschäftsordnung oder eines Operationsstatuts, deren Einhaltung durch die Lenkungsgruppe durchgesetzt wird, ist dafür Grundvoraussetzung.

- Die Unterstellung der Operationsabteilung unter einem Operationsmanager löst den Operationsbetrieb aus dem direkten Verantwortungsbereich der Kliniken aus. Rasche Entscheidungswege werden möglich. Unterschiedliche Stellenprofile und Professionskultur bergen Konfliktpotential. Eine isolierte Betrachtung des Operationssaalbetriebes birgt jedoch die Gefahr einer Vernachlässigung der Wertschöpfungskette. 
Diese Arbeit möchte nicht die Pro's und Contra's einzelner Organisationsformen vertiefen. Es ist aber sinnvoll, bei der Planung von effizienzsteigernden Maßnahmen im Operationsprozess die Geschwindigkeit des damit verbundenen Kulturwandels mit zu berücksichtigen. Ein Vorgehen mit Ablaufoptimierungen innerhalb des Lösungsansatzes 1 ist ebenso denkbar, wie ein stufenweises Übergehen von Lösungsansatz 1 über 2 auf Lösungsansatz 3.

Bei von allen Berufsgruppen akzeptierten Instrumenten zur Monitorisierung des Operationsprozesses z. B. durch eine Operationslenkungsgruppe (Disposition, Ist) Soll-Analyse, Geschäftsordnung) wird eine kontinuierliche Organisationsentwicklung ermöglicht. Im Folgenden wird die schrittweise Implementierung des Veränderungsprozesses, wie er an einem Haus der Maximalversorgung erfolgte, erläutert [3,4,5].

\section{Implementierung einer Operationsmanagementgruppe (OPMG)}

Das OP-Management ist mehr als die tägliche Koordination der Abläufe in den OPSälen.

Es bedeutet interdisziplinäre Zusammenarbeit mit aktivem Schnittstellenmanagement, das weit in das Vorfeld und in die Folgebereiche einer OP-Abteilung reichen muss.

Um im OP effiziente Arbeitsabläufe gewährleisten zu können, muss ein aktives OP-Management die nachfolgende Aufgaben mitgestalten:

- Der erster Auftrag der Lenkungsgruppe beinhaltet das Formulieren der klinikadaptierten strategischen Leitlinien. Eine Vernehmlassung/Freigabe soll über alle Kliniken und Fachbereiche durchgeführt werden. Die OPMG ist zuständig für die Organisationsform, die strategischen Vorgaben und alle diesbezüglichen generellen Rahmenbedingungen wie $z$. B. das spätere Controlling.

- Eine Formierung einer Projektgruppe ist sinnvoll. Die Projektgruppe fokussiert auf einen Auftrag und die strategischen Leitlinien und teilt die verschiedenen Aufgaben in Projektabschnitten den Mitgliedern zu. Bereits in der Startphase des Projektes werden die kritischen Erfolgsfaktoren (KEF's) formuliert und im Projektplan einbezogen.
- Basierend auf einer Ist-Analyse werden neue Soll-Prozesse beschrieben. Die Soll-Prozesse können besonders für die OP-Planung durch Planungssoftware unterstützt werden. Prozessvarianten werden mittels geeigneter Software simuliert.

- Die Erstellung und Umsetzung eines interdisziplinären OP-Statuts (Geschäftsordnung), dass die Zusammenarbeit der verschiedenen Berufssparten verbindlich regelt.

- Die Schaffung eines Koordinationszentrums im OP mit der Aufgabe, Programmablauf und notfall- oder planungsbedingte Programmänderungen zu koordinieren). Leitlinie dafür ist das OP-Statut.

- Controlling $[4,5]$

\section{Leitlinien}

Die Leitlinien für eine chirurgischen Versorgungseinheit müssen konform mit den strategischen Leitplanken des Gesamtspitals sein.

Vorhandene Leitbilder und eine formulierte Strategie ermöglichen eine einheitliche Ausrichtung für das gesamte Dienstleistungsunternehmen.

\section{Bestandesaufnahme/OP-Simulation/ Soll-Prozesse}

\section{Ist-Analyse}

Eine Ist-Analyse muss den aktuellen OPProzess als ganzes abbilden. Die einzelnen kritischen Prozess-Schritte müssen speziell berücksichtigt werden. Die OPAnmeldung pro Klinik resp. Fachbereich, Notfallmanagement (Kategorisierung der Notfälle, aber auch deren Anmeldung sowie Regeln zur Abwicklung von Notfällen), OPS-Reglement, Disposition, Supervising, Organisations-Struktur, Führungsprozesse usw. Siehe $\mathbf{A b b . 1}$ als Beispiel für den Ablauf eines Operationsprozesses.

\section{OP-Simulation}

Die Simulation mit entsprechender Software wird auf der Basis des Ist-Modells anhand getroffener Annahmen und Erwartungen bezüglich Prozessgestaltung, Kapazitäten und Ressourcenbedarf dynamisch visualisiert. Prüfbare Resultate werden aufgezeichnet und grafisch dargestellt. Das Modell kann eine strategische Planung der OP-Prozesse ermöglichen. Dabei zeigt sich vor allem im Ausbildungsspital die Problematik der unterschiedlichen Ausbildungsstufen von An- ästhesisten und Operateuren. Eine Kategorisierung der Behandlungsgeschwindigkeiten für den gleichen Eingriff durch verschiedene Ärzte (Assistent, Facharzt, Oberarzt, Chefarzt) kann zur Verbesserung einer homogenen Planung führen. Dies kann Wartezeit und Blockierung und damit Effizienzverlust im Behandlungsprozess reduzieren. Optimales Planen wird dadurch verbessert, bleibt im Alltag aber immer noch stark abhängig von anderen Faktoren, wie Standardisierbarkeit und anatomiebedingte lokale $\mathrm{Pa}$ tientenverhältnisse.

Die Ist-Analyse und die Simulation zeigt als Resultat die typischen Probleme auf, wie: ungenügende Saalauslastung, Überkapazitäten, unpünktlicher Op-Beginn, lange Wechselzeiten und über die verordnete Arbeitszeit reichende Operationsprogramme (Abb.2). Dargestellt sind die Anzahl der Patienten, die sich während der regulären Betriebszeiten im OP-Trakt befinden $[1,6]$.

\section{Soll-Prozesse}

Im Zuge der Ausformulierung der SollProzesse können Indikatoren entwickelt werden, die das OP-Controlling unterstützen. Mögliche Indikatoren können abgeleitet werden von: Anfangszeit, Wechselzeit, Schlusszeit, Abweichung gegenüber der Planung, Anzahl der Notfälle, usw.

\section{OP-Statut (Geschäftsordnung)}

Das OP-Statut beinhaltet fachübergreifend schriftlich festgelegte Regeln. Für die OP-Programmplanung, die Kontrolle und die Umsetzung des OP-Plans und die aus dem OP-Plan resultierende Ablauforganisation eignen sich 3 verschiedene Planungsebenen:

Patientenebene: Alle den Patientenbetreuenden Leistungsbereiche wie die operativen Fachdisziplinen inkl. Anästhesie (Prämedikation), die Bettendisposition, die Tageschirurgie, die Ambulatorien und Sprechstunden

- Organisation/Kontroll/Lenkungsebene: z.B. OP-Lenkungsgruppe (OPMG)

- Operationsprozessebene: OP-Koordination inkl. Supervising und die ausführenden Berufsgruppen innerhalb der OPS. (Abb. $\mathbf{3}$ ).

Die Berücksichtigung dieser Planungsebenen macht eine koordinierte Einbeziehung aller Beteiligten möglich. Ein möglichst reibungsloser Programmablauf 


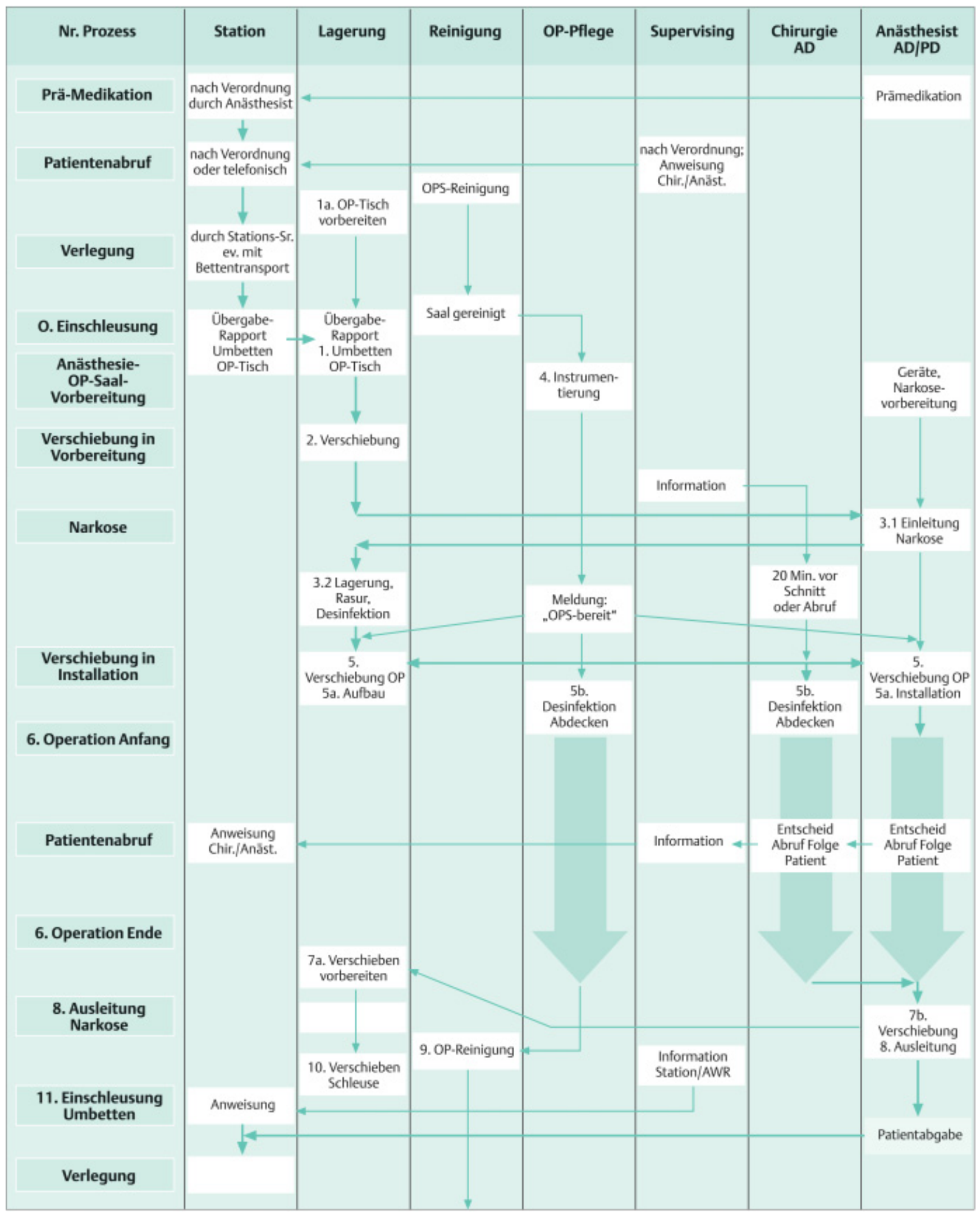

Abb.1 Ablauf eines Operationsprozesses. 


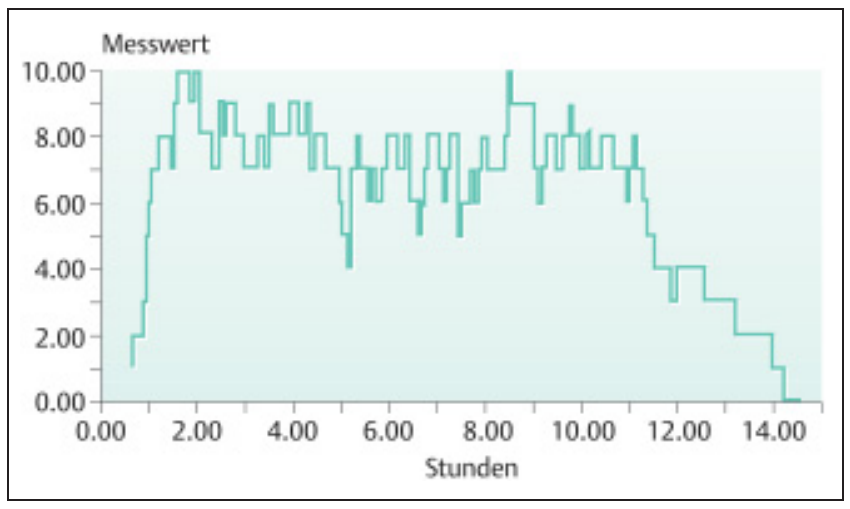

Abb. 2 Simulierte OP-Auslastung unter Berücksichtigung der verfügbaren Ressourcen und Patienmix.

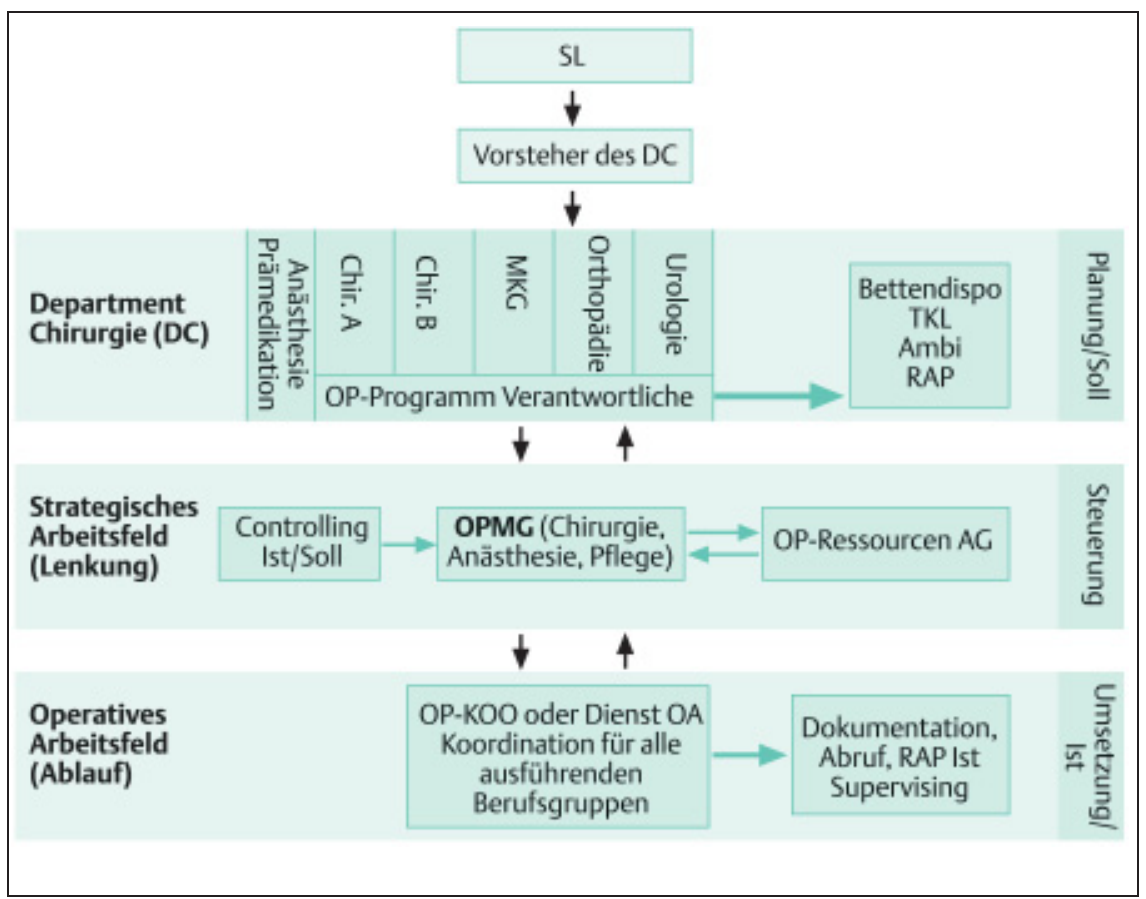

Abb. 3 Darstellung der 3 OP-Planungsebenen.

wird so ermöglicht. Das OP-Statut sollte folgende Elemente enthalten:

- Definition der strategischen Leitlinien

- Spielregeln für die OP-Planung und den OP-Betrieb

- Rahmenbedingungen der Umsetzung und Überprüfung der strategischen Leitlinien $[1,4]$.

\section{OP-Koordination}

Die tägliche Disposition eines OP-Bereiches richtet sich nach den Leitlinien der OPMG. Ein Mitglied der OPMG kann selber mit dieser Aufgabe betraut werden oder die tägliche Disposition delegieren. Die OP-Koordination ist ebenfalls verantwortlich für die Disposition von allenfalls verschobenen Wahloperationen. Sie ist somit die Drehscheibe für das Tagesgeschäft mit dem Ziel, die zur Verfügung stehende OPSKapazität optimal zu nutzen. Nachfolgend formieren. Bei Programmänderungen und insbesondere bei Notfällen ist eine verbindliche Regelung des Informationsflusses erforderlich. So werden beteiligte Entscheidungsträger und involvierte Berufsgruppen zeitgerecht kontaktiert und informiert. Das OP-Statut enthält interdisziplinär vereinbarte Regeln, wie eine verbindliche Kategorisierung der Notfälle und wie im laufenden Programm dringliche Notfälle abgearbeitet werden. Die OPKoordination stützt sich dabei auf das OPStatut und berichtet periodisch an die OPMG resp. an den OP-Manager $[3,4,5]$.

\section{Administration}

Die Planung des OP-Programms wird durch die operativen Fachdisziplinen durchgeführt. Sie orientiert sich an der medizinischen Dringlichkeit und den notwendigen Ressourcen, ebenso sind die Betten-, die Intensiv- und Aufwachraumkapazität zu berücksichtigen.

Die OP-Planung wird in einen Grob- und einen Feinplanungsprozess aufgeteilt. Der Grobplanungsprozess wird durch den Arztdienst gestartet und kann wie folgt organisiert werden:

Alle Beteiligten erhalten OP-Slots oder OPKapazität. Der Arztdienst kann nach Bedarf für kurzfristige OP-Anmeldungen über Vorhalteslots planen. Die Bettendispo plant lang- bis mittelfristig über Elektiv-Slots. Notfalleingriffe werden über Notfall-Slots angemeldet und können so durch die OPKoordination elektronisch disponiert werden. Ferienabwesenheiten oder sonstige Abwesenheiten einzelner Fachdisziplinen müssen rechtzeitig gemeldet werden, ansonsten wird die OP-Kapazität vergeben. Das Softwaretool ermöglicht das Ausdrukken von provisorischen OP-Plänen wochen- oder auch monatsweise. Für die Feinplanung sind die Programmverantwortlichen der Kliniken selber verantwortlich. Sie legen spezifisch ihre Reihenfolge der OP-Eingriffe fest oder führen letzte Änderungen durch. Anschließend wird am Tag vorher ein abschließender Rapport den definitiven OP-Plan festlegen (Soll-Plan). Nach wie vor ermöglicht das System auch nicht planmäßige Änderungen. Die OP-Koordination wird am OP-Tag entsprechend informiert und kann die Änderungen berücksichtigen. Es ist sinnvoll, wenn man den administrativen OP-Anmeldungsprozess definiert und schriftlich festlegt. Alle OP-Eingriffe werden im OP-Saal entsprechend definierten Kategorien zeitlich registriert (Patient im Saal, Schnitt/Nahtzeiten, Saal frei und Patientenabruf) und elek- 


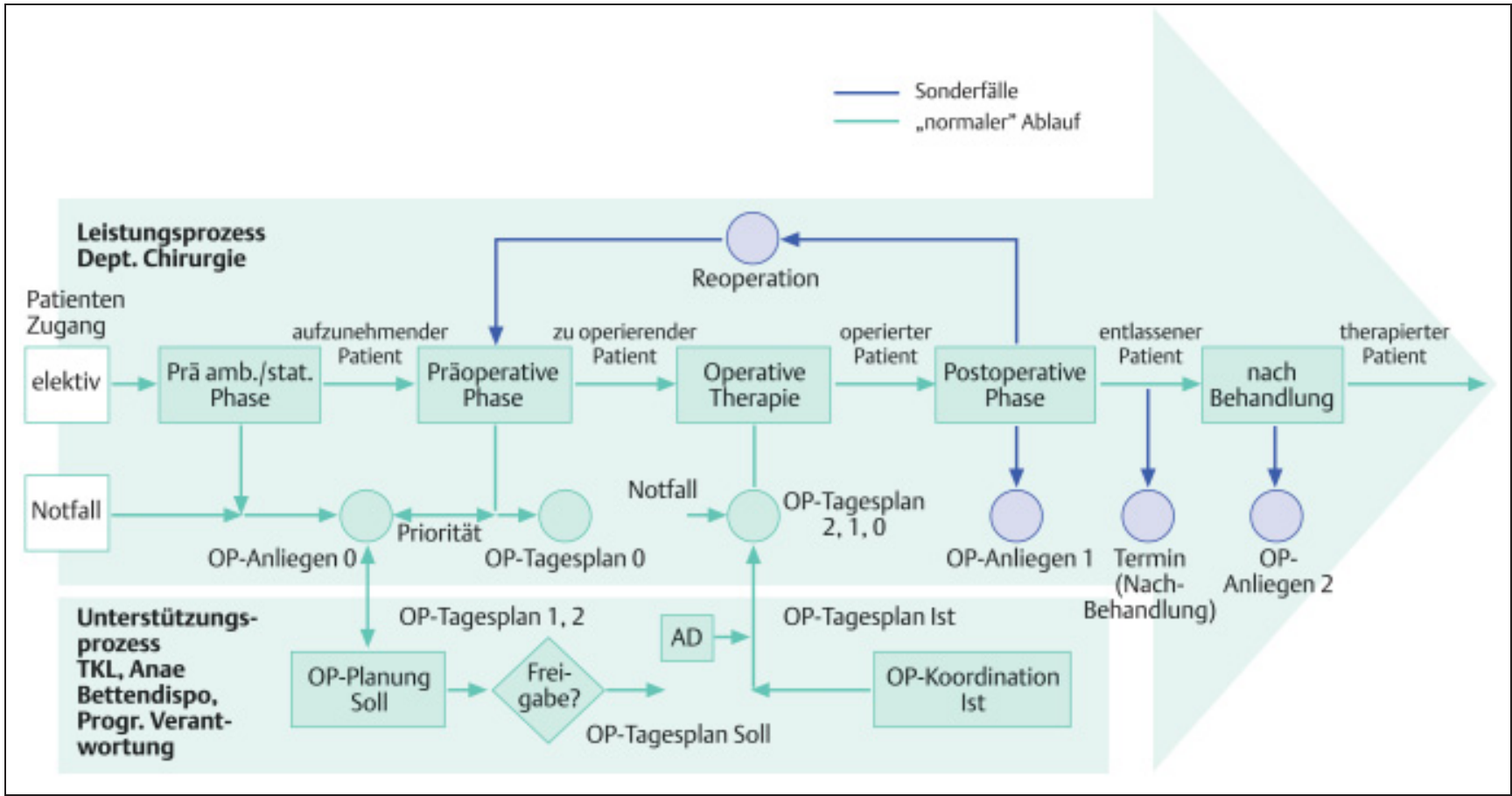

Abb.4 Referenzmodell eines Patientendurchlaufs bei operativer Therapie (Ist/Soll).

tronisch abgelegt (Ist-Plan). Ein Ist/SollVergleich wird periodisch ausgewertet und kann den Entscheidungsträgern zur Verfügung gestellt werden. Siehe $\mathbf{A b b . 4}$ Referenzmodell eines Patientendurchlaufs bei operativer Therapie (Ist/Soll).

\section{OP-spezifisches Controlling}

Die OPMG beruft regelmäßig Leistungssitzungen mit weisungsbefugten Vertretern aller im OP tätigen Abteilungen/Kliniken/Fachbereiche ein. Ziel ist es, die Einhaltung der Geschäftsordnung und Zielparameter zu präsentieren [3].

Die ausgewiesenen Defizite gilt es interdisziplinär zu diskutieren, um eine weitere Verbesserung und Transparenz der Abläufe und damit der Wirtschaftlichkeit und der Patienten/Mitarbeiterzufriedenheit zu erreichen.

\section{Schlussfolgerung}

Veränderungen im Operationsbereich zum Zweck der Effizienzsteigerung erfordern klare, an die Strategie des Spitalträgers adaptiere Leitlinien. Integrierte, alle Leistungsträger berücksichtigende Patientenpfade für die präoperative, die operative und die postoperative Phase regeln wichtige Schnittstellen und vereinfachen die interne und die externe Kommunikation. In einem Zentral-OP treffen unterschiedliche Interessen aufeinander.
Kliniken und Institute können nach unserer Erfahrung in einem partizipativen und schrittweisen Prozess erfolgreich eingebunden werden, wenn der Kommunikation die nötige Beachtung geschenkt wird.

Gemeinsame Planungswerkzeuge erlauben dabei eine bessere Koordination und Ressourcennutzung der einzelnen Kliniken. Auf der Grundlage einer IstAnalyse, welche die verschiedenen Kategorien von Erfahrungsstufen der Anästhesisten und der Operateure berücksichtigt, lässt sich der Operationsprozess simulieren und Soll-Ziele lassen sich realistisch definieren. Die Monitorisierung des Operationsprozesses durch die elektronische Erfassung von definierten Fixpunkten ermöglicht datenbasierte schrittweise und zielkonforme Veränderungsprozesse. Das Primat der Patientensicherheit, der Patientenzufriedenheit und der Mitarbeiterzufriedenheit sollte trotz betriebswirtschaftlichem Fokus nachhaltig verfolgt werden. Die Geschwindigkeit und die Nachhaltigkeit des Veränderungsprozesses zur Optimierung des Operationsprozesses kann durch die Verbesserung einzelner Prozess-Schritte, durch Bestimmung einer interdisziplinären Operationsmanagementgruppe, welche die Instrumente zur Prozessregelung und zum Prozessmonitoring interdisziplinär erarbeitet, oder durch die Wahl eines Operationssaal Managers moduliert werden [7,8].

\section{Literatur}

1 Geldner G, Eberhart LHJ, Trunk S, Dahmen KG, Reissmann T, Weiler T, Bach A. Effizientes OP-Management. Anästhesist 2002; 51 : $760-767$

2 Herfarth Ch. „Schlanke“ Chirurgie. Chirurg 2002; 73: 103-04

3 P. Wigger Modernes Management der Operationsabteilung am Kantonsspital Winterthur. Schweizerische Ärztezeitung 2001; 82: Nr. 4

4 Riedl S. Modernes Operationsmanagement im Workflow Operation. Chirurg 2002; 73: $105-110$

5 Motsch J, Martin E. Beitrag der Anästhesie zum Workflow Operation. Chirurg 2002; 73: $118-121$

6 Friess H, Kleeff J, Hartwig W, Schmidt J, Radnic S, Auer S, Büchler MW. Zentrales Patientenmanagement in der Chirurgie. Chirurg 2002; 73: $111-117$

7 Gfrörer R, Schüpfer G. Das Operationssaalteam. ZfO 2004; 6: 333-339

8 Grahmann R. Konflikte im Krankenhaus; ihre Ursachen und ihre Bewältigung im pflegerischen und ärztlichen Bereich, Bern 1996: 21.

\section{Dipl.-Ing. Physik Theo H. M. Smits} Qualitätsmanager

Dr. med. Guido Schüpfer, MBA HSG Leitender Arzt Anästhesie

Priv.-Doz. Dr. med. Reto Babst

Vorsteher Departement Chirurgie Chefarzt Unfallchirurgie

Kantonsspital Luzern Spitalstrasse

CH-6000 Luzern 16 